$\Rightarrow$ SKIN CANCER

\title{
BRAF+MEKi and ICl triplets show promise in melanoma
}

64

These data

provide

the first

demonstration

of the safety

and clinical

benefit of

combining two

oncogene-

targeted

therapies with

an $\mathrm{ICl}$

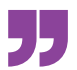

BRAF and MEK inhibition (BRAF+

MEKi) and immune-checkpoint inhibition (ICI) have each drastically improved the outcomes of patients with metastatic melanoma. BRAF+ MEKi (for those with $B R A F^{\mathrm{V} 600}$-mutant disease) results in higher objective response rates (ORRs), whereas ICI typically results in more-durable responses. Importantly, these treatments have distinct mechanisms of action and thus considerable interest has been placed on combining them. Now, results from three early phase clinical trials in patients with previously untreated metastatic $B R A F^{\mathrm{V} 600}$-mutant melanoma highlight the promise of this strategy.

First, Ryan Sullivan et al. conducted a phase Ib study in which 39 patients received the BRAF inhibitor vemurafenib and the MEK inhibitor cobimetinib combined with the anti-programmed cell death 1 ligand 1 (PD-L1) antibody atezolizumab. Second, in a phase I portion of the KEYNOTE-022 study, Antoni Ribas et al. treated 15 patients with the anti-programmed cell death 1 (PD-1) antibody pembrolizumab together with the BRAF inhibitor

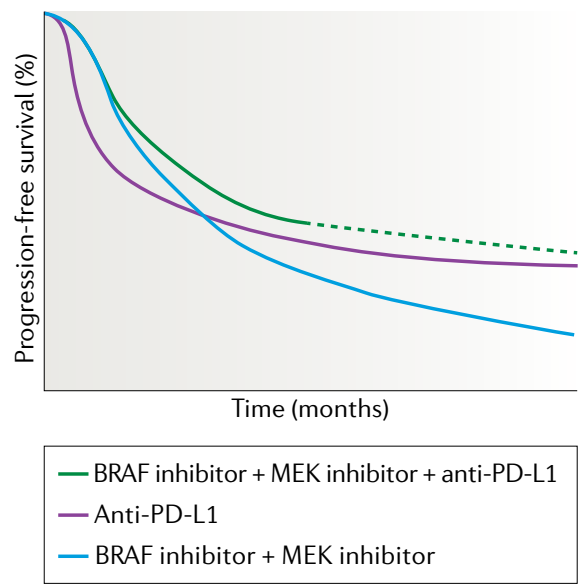

Credit: Image adapted from Rozeman, E. A. \& Blank, C. U. Nat. Med. 25, 879-882 (2019), Springer Nature Limited dabrafenib and the MEK inhibitor trametinib. Finally, in a randomized phase II part of KEYNOTE-022, 120 patients received dabrafenib and trametinib plus either pembrolizumab or placebo.

The efficacy data obtained from these three studies were remarkably consistent and reveal ORRs with the triplet $\mathrm{BRAF}+\mathrm{MEKi}$ plus ICI of $72 \%, 73 \%$ and $63 \%$ (compared with $72 \%$ with BRAF+MEKi plus placebo in the phase II trial), respectively. The respective complete response rates were $21 \%$, $7 \%$ and $18 \%$ (13\% with placebo). The median progression-free survival durations were 12.9 months, 15.4 months and 16.0 months (10.3 months with placebo).

"These data provide the first demonstration of the safety and clinical benefit of combining two oncogene-targeted therapies with an ICI," says Ribas. "Subgroup analysis of our phase II trial also showed a better outcome with the triplet in patients with a performance status of 1 or elevated serum lactate dehydrogenase levels," patients who typically have a poor prognosis.

Interestingly, "on the basis of a limited number of tumour biopsy samples, we saw increases in $\mathrm{CD}^{+}$ $\mathrm{T}$ cell infiltration in most patients following a 28-day BRAF+MEKi run-in period," Sullivan explains. These finding are in keeping with immunomodulatory effects of BRAF+MEKi and potentiation of ICI observed in preclinical models. Accordingly, Ribas et al. observed increased tumour infiltration of $\mathrm{CD}^{+} \mathrm{T}$ cells, as well as expression of PD-L1 and a T cell-inflamed gene signature, after triplet therapy.

The incidence of adverse events (AEs) across all three studies was higher than that expected for either
BRAF + MEKi or ICI alone. Grade $\geq 3$ treatment-related AEs occurred in $58-73 \%$ of patients (versus $27 \%$ of the placebo arm of KEYNOTE-022), most commonly increased serum levels of liver enzymes, fever and hypophosphataemia. Notably, $6.7 \%$ of patients who received pembrolizumab in the phase II trial developed grade $\geq 3$ pneumonitis, with one fatality. "Whether modifications to the regimen (lead-in with BRAF+MEKi, intermittent $\mathrm{BRAF}+\mathrm{MEKi}$, and so on) will enable patients to better tolerate treatment will be crucial to determine; in particular, the ORR data from the randomized portion of the KEYNOTE-022 study suggest that efficacy is less than it could be owing to toxicities," Sullivan states.

"Our work establishes important data on which to build; the logical next step is the read out of two phase III trials that have completed accrual, the TRILOGY study of the atezolizumab regimen (NCT02908672) and the COMBI-i study of dabrafenib, trametinib and the anti-PD-1 antibody spartalizumab (NCT02967692)," Sullivan continues. "However, what is clear is that responses seem deeper with the triplet regimens, suggesting that we might be triggering a more robust response in some patients," he concludes. "Further data from the ongoing phase III studies will also give us more information about whether such strategies might be an option for all patients or just for patients with poor prognostic factors who derive limited benefit from the individual therapies," adds Paolo Ascierto, who was also involved in the phase II trial.

David Killock

ORIGINAL ARTICLE Sullivan, R. J. et al. Atezolizumab plus cobimetinib and vemurafenib in BRAF-mutated melanoma patients. Nat. Med. 25, 929-935 (2019) | Ribas, A. et al. Combined BRAF and MEK inhibition with PD-1 blockade immunotherapy in BRAF-mutant melanoma. Nat. Med. 25, 936-940 (2019)| Ascierto, P. A. et al. Dabrafenib, trametinib and pembrolizumab or placebo in BRAF-mutant melanoma. Nat. Med. 25 , 941-946 (2019) 